\title{
Textbook outcome after major hepatectomy for perihilar cholangiocarcinoma - definitions and influencing factors
}

\author{
Christian Benzing ${ }^{1}$ (D) Lena Marie Haiden ${ }^{1} \cdot$ Felix Krenzien $^{1} \cdot$ Alexa Mieg $^{1} \cdot$ Annika Wolfsberger $^{1}$. \\ Cecilia Filiz Atik ${ }^{1}$ - Nora Nevermann ${ }^{1}$ - Uli Fehrenbach ${ }^{2}$ - Wenzel Schöning ${ }^{1} \cdot$ Moritz Schmelzle $^{1}$. \\ Johann Pratschke ${ }^{1}$
}

Received: 9 November 2021 / Accepted: 9 February 2022 / Published online: 4 March 2022

(c) The Author(s) 2022

\begin{abstract}
Purpose The concept of "textbook outcome" (TO) as composite quality measure depicting the ideal surgical has not yet been defined for patients undergoing major hepatectomy (MH) for perihilar cholangiocarcinoma (PHC). This study sought to propose a uniform definition through a systematic literature review as well as to identify patient- or procedure-related factors influencing TO.

Methods In this retrospective study, we analyzed all patients undergoing MH for PHC at our department between January 2005 and August 2019. After conducting a systematic literature search, we defined TO as the absence of 90-day mortality and major complications, no hospital readmission within 90 days after discharge, and no prolonged hospital stay $(<75$. percentile). A binary logistic regression analysis was performed to identify factors influencing TO.

Results Of 283 patients, TO was achieved in 67 (24\%) patients. Multivariate analysis revealed that preoperative biliary drainage was associated with a decreased $(\mathrm{OR}=0.405,95 \% \mathrm{CI}$ : $0.194-0.845, p=0.016)$ and left-sided-resection $(\mathrm{OR}=1.899$, 95\% CI: 1.048-3.440, $p=0.035$ ) with increased odds for TO. Overall survival (OS) and DFS (disease-free survival) did not differ significantly between the outcome groups (OS: $p=0.280$, DFS: $p=0.735$ ). However, there was a trend towards better overall survival, especially in the late course with TO.

Conclusion Our analysis proposed a uniform definition of TO after MH for PHC. We identified left hepatectomy as an independent factor positively influencing TO. In patients where both right- and left-sided resections are feasible, this underlines the importance of a careful selection of patients who are scheduled for right hepatectomy.
\end{abstract}

Keywords Textbook outcome $\cdot$ Perihilar cholangiocarcinoma $\cdot$ Major hepatectomy

Christian Benzing and Lena Marie Haiden contributed equally to this work and share first authorship.

Moritz Schmelzle and Johann Pratschke contributed equally to this work and share senior authorship.

Moritz Schmelzle

moritz.schmelzle@charite.de

1 Department of Surgery, Campus Charité Mitte I Campus Virchow-Klinikum, Experimental Surgery and Regenerative Medicine, Charité - Universitätsmedizin Berlin, Augustenburger Platz 1, 13353 Berlin, Germany

2 Department of Radiology, Charité - Universitätsmedizin Berlin, Berlin, Germany

\section{Introduction}

Perihilar cholangiocarcinoma (PHC) is a rare malignant tumor arising from the bile duct that is characterized by a poor prognosis [1]. So far, major hepatectomy $(\mathrm{MH})$ is the only established, potentially curative treatment for patients with PHC. Radical surgical approaches such as hilar en bloc resection described by Neuhaus and colleagues were able to increase overall survival rates in the last years [2, 3]. However, high postoperative morbidity and mortality still remain unsatisfactory, despite advances in preoperative workup such as portal vein embolization (PVE) and improvements in perioperative management [4-6]. Diagnosis at an advanced stage, which is often accompanied by bile duct obstruction, cholestasis, cholangitis, and poor liver function, and radical surgical approaches are associated with morbidity 
rates as high as $50-60 \%$ and substantial mortality rates ranging between 5 and $18 \%$, even in high-volume centers [5, 7-11]. After resection, common complications are bile leakage, septic, or vascular complications; however, posthepatectomy liver failure (PHLF) being associated with a high associated mortality is feared the most $[7,12]$.

Recently, the concept of the "textbook outcome" (TO) as composite quality measure depicting the ideal surgical outcome has been used especially in complex oncological surgery [13-16]. TO is achieved when certain predefined desirable outcomes are simultaneously present in a patient's postoperative course representing a much more comprehensive summary of a patient's hospitalization than one singular outcome parameter such as mortality [17]. There is no generally accepted definition of TO and different parameters are used to define TO depending on the type of surgery or tumor [18]. Traditional quality measures like postoperative mortality (e.g., 90-day mortality), postoperative morbidity, or length of stay (LOS) can often be found in the definition of TO, but histopathological factors such as the presence of an R0 resection are increasingly used as well [13, 19, 20]. As a comprehensive quality measure, TO can not only be used by patients and health care providers for assessing the quality of surgical care or hospital performance but also by surgeons to optimize preoperative workup and surgical care [19]. TO has not been established in PHC surgery yet and TO rates as well as potentially influenceable patientand procedure-related factors TO are unknown. As it might help improve surgical management and thus postoperative morbidity and mortality, this study was conducted to define TO after major liver resection for PHC, identify prognostic factors predicting $\mathrm{TO}$, and analyze the impact of achieving a TO on overall survival (OS) and disease-free survival (DFS).

\section{Methods}

\section{Patients and study design}

Patients undergoing MH in curative intent for PHC between January 2005 and August 2019 at the Department of Surgery, Campus Charité - Mitte and Campus Virchow Klinikum, Charité - Universitätsmedizin Berlin were retrospectively analyzed. This retrospective study was approved by the local ethics committee (EA2/006/16 and EA1/358/16). Variables included in the analysis were general patient characteristics such as gender, age, American Association of Anesthesiologists (ASA) score, and body mass index (BMI). Perioperative and histopathological data were recorded as well as data on overall survival (OS) and disease-free survival (DFS). Postoperative morbidity as classified by Dindo-Clavien during hospitalization, 30- and 90-day mortality, length of hospital stay, and intensive care unit stay, respectively, were recorded as well [21].

\section{Preoperative management}

All patients who were referred to our institution for surgical treatment underwent a highly individualized and detailed workup. This routinely included computed tomography and/ or magnetic resonance imaging of the chest and abdomen as well as endoscopic retrograde cholangiography (ERC). Biliary drainage with ERC and biliary stenting or percutaneous transhepatic cholangiodrainage (PTCD) was performed when necessary. Routinely, Carbohydrate antigen 19-9 (CA 19-9) was measured before resection. On patients with suspected peritoneal dissemination, diagnostic laparoscopy or laparotomy was performed.

\section{Surgical procedure}

All patients who underwent $\mathrm{MH}$ for PHC were included in the analysis. The surgical resection was performed either as a right or left sided major hepatectomy with extrahepatic bile duct resection as described before [2,3]. Biliary reconstruction was performed as end-to-side hepaticojejunostomy. Patients with extrahepatic bile duct resection alone or multivisceral resections, e.g., hepatoduodenopancreatectomy (HPD) were excluded from the analysis as well as patients with intrahepatic or distant metastases or local peritoneal carcinomatosis.

\section{Histopathology}

In all cases, PHC was confirmed according to the histopathological reports of the resected specimen. Furthermore, data on resection and lymph node status as well as perineural sheath infiltration, microvascular infiltration, lymphangiosis carcinomatosa, and tumor differentiation were collected from pathology reports. Based on the collected data and the TNM classification valid at the time of resection, patients were assigned to the appropriate tumor stage according to the Union for International Cancer Control (UICC, 7th edition).

\section{Textbook outcome and postoperative course}

As TO had not yet been defined for major liver resection for PHC, a systematic literature review was conducted to evaluate common TO definitions used in HPB surgery. TO was defined based on the results of this literature review and common complications after PHC resection mentioned in the established literature. The search terms "textbook outcome" and "textbook oncologic outcome" were shortened to "textbook outcom*" and "textbook oncologic 
outcom*" and furthermore combined with the Boolean operator AND the following search terms: "liver surgery," "hepatectomy," "resection of liver," "pancreatic surgery," "pancreaticoduodenectomy," "resection of pancrea*," "hepatopancreatic surgery." The publication period was limited to the years 2010 to 2020. Only studies in English with TO as primary endpoint were considered. Study designs such as reviews or meta-analyses were excluded. In addition, studies that investigated hepatic resections performed laparoscopically were excluded. TO was defined as the absence of 90-day mortality and major complications (i.e., > grade II according to Dindo-Clavien), no hospital readmission within 90 days after discharge, and no prolonged hospital stay (i.e., $<75$. percentile). The dichotomous textbook outcome was achieved when all four abovementioned individual criteria were observed in one patient after resection. Patients that could not be classified as either TO or NTO due to missing data were excluded from the analysis.

\section{Follow-up}

Patients were followed up in the outpatient clinic or with their general practitioner. Check-ups routinely included testing of CA 19-9 serum levels and abdominal ultrasound, $\mathrm{CT}$, or MRI. Whether adjuvant chemotherapy was performed was recorded as well.

\section{Statistics}

Statistical analysis was performed using IBM SPSS Statistics for Macintosh Version 25.0 (IBM Corp., Armonk, NY, USA). A $p$-value $<0.05$ was considered statistically significant. Continuous parameters are presented as median and range and statistically compared with the non-parametric Mann-Whitney- $U$-test. Categorical data are displayed as counts and percentages and are compared using the chisquared test or Fisher's exact test, when necessary. To identify independent factors influencing TO, a binary logistic regression analysis was performed. Results are reported as odds ratio (OR) and 95\% confidence interval (95\% CI). Prognostic factors were included in the regression model when a significant influence on TO was detected in univariate analysis. The variables age and gender were included in multivariate analysis regardless of significance. Survival was estimated with the Kaplan-Meier method and compared between the outcome groups with the log-rank test. Patients who died within 90 days of surgery were not included in the survival analyses. A subgroup analysis was performed with all patients surviving at least 30 months.

\section{Results}

\section{Definition of TO}

A total of 20 records were identified through database searching. After removal of duplicates, 12 records were screened for eligibility. After excluding sources that did not meet the inclusion criteria, a total of 7 studies in HPB surgery with TO as primary endpoint were identified for the final review. Figure 1 summarizes the search algorithm, whereas Table 1 and Table 2 give a summary on included studies. All studies were retrospective and multicenter studies that were published between 2019 and 2020 . The smallest and largest series contained 687 and 21234 patients, respectively. Postoperative mortality, length of stay, and hospital readmission were part of the TO definition in all seven studies. Four records [17, 19, 20, 24] used 30-day mortality and 30-day readmission, whereas three studies [13, 22, 23] used a span of 90 days instead. Histopathological criteria were used in four studies [13, 17, $19,20]$ to define TO. This included tumor-free resection margins in all four studies [13,17, 19, 20] and absence of lymph node metastases in one [20] study. Adjuvant chemotherapy and the need for postoperative transfusion were part of the TO definition in two studies [17, 20]. One study was not able to get detailed information on postoperative morbidity and used LOS instead [20]. Specific complications such as bile leakage or postoperative pancreas fistula were part of the TO definition on one study [24]. All studies investigated TO rates for different tumor entities or HPB procedures, and most studies sought to identify TO-influencing factors after surgery (Table 2).

\section{Patients' characteristics}

In this study, two hundred and eighty-three patients who underwent surgical resection for PHC in curative intent between January 2005 and August 2019 and met the inclusion criteria were analyzed. The cohort comprised $171(60 \%)$ male and 112 (40\%) female patients with a mean age of 65 (33-86) years. The majority of patients was grouped in either ASA $2(56 \%, n=159)$ or ASA 3 $(38 \%, n=107)$, thus suffering from pre-existing conditions. Table 3 summarizes all patient characteristics of the total cohort. The majority of patients presented with Bismuth Type IV PHC or advanced UICC stages (IIIB-IVA: 48\%, $n=134)$. Tumor-free resection margins were achieved in $68 \%(n=189)$ of all patients, whereas $48 \%(n=132)$ patients had histopathologically confirmed local lymph node metastases. Most patients presented with a moderate tumor differentiation (G2: $68 \%, n=190)$. A total of 
Fig. 1 Flowchart of search algorithm

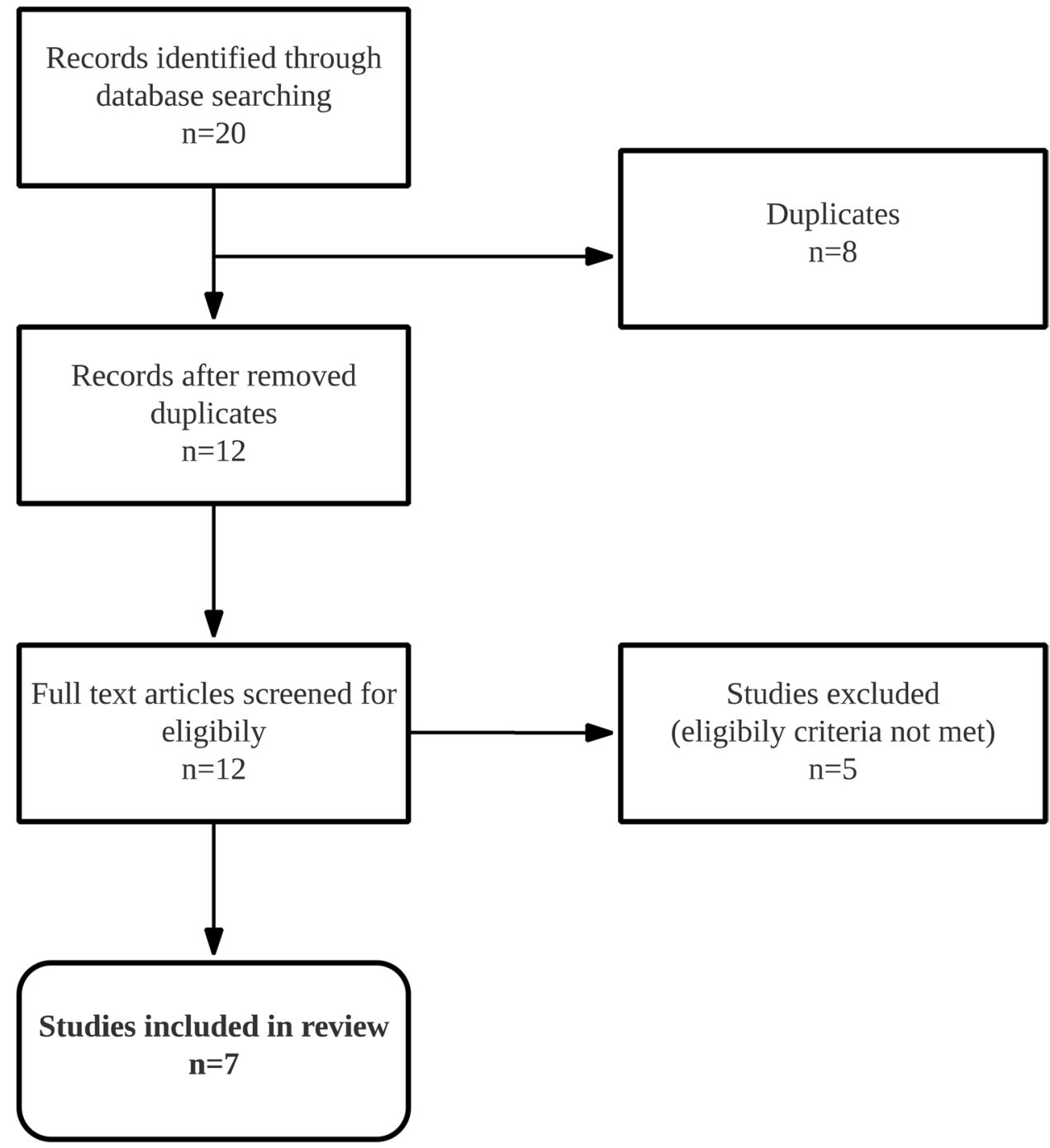

Table 1 TO studies included in review

\begin{tabular}{llllll}
\hline Author & $\begin{array}{l}\text { Year of } \\
\text { publication }\end{array}$ & Study characteristics & Year of inclusion & $\begin{array}{l}\text { Number of } \\
\text { patients }\end{array}$ & TO definition used \\
\hline Heidsma et al. [13] & 2020 & Retrospective, multicenter & $2000-2016$ & 821 & LOS, RA90, 90DM, R0, PK<III \\
Mehta et al. [22] & 2020 & Retrospective, multicenter & $2013-2017$ & 21234 & LOS, RA90, 90DM, PK0 \\
Merath et al. [17] & 2019 & Retrospective, multicenter & $1993-2015$ & 687 & LOS, 30DM, RA30, R0, PK0, Tr0 \\
Merath et al. [23] & 2020 & Retrospective, multicenter & $2013-2015$ & 13467 & LOS, RA90, 90DM, PK0 \\
Sweigert et al. [20] & 2020 & Retrospective, multicenter & $2006-2015$ & 18608 & LOS, RA30, 30DM, aCT, N0, R0 \\
Tsilimigras et al. [19] & 2020 & Retrospective, multicenter & $2005-2017$ & 1829 & LOS, RA30, 30DM, R0, PK<III \\
Van Roessel et al. [24] & 2020 & Retrospective, multicenter & $2014-2017$ & 3341 & LOS, RA30, 30DM, BL, PK<III, \\
& & & & & PPF, PPH
\end{tabular}

TO textbook outcome, RA30 no 30-day readmission, LOS no prolonged hospital stay, 30DM no 30-day mortality, RA90 no 90-day readmission, 90DM no 90-day mortality, $P K O$ no postoperative complications according Dindo-Clavien [21], $P K<I I I$ no major postoperative complications according Dindo-Clavien (i.e., grade <III) [21], $R O$ tumor-free resection margin after resection, $T r 0$ no perioperative blood transfusion, $P P F$ no postoperative pancreas fistula, $B L$ no bile leakage, $P P H$ no post-pancreatectomy hemorrhage, $a C T$ adjuvant chemotherapy, $N O$ absence of lymph node metastasis 
Table 2 TO studies included in review

\begin{tabular}{|c|c|c|c|}
\hline Author & Tumor entity & Procedure & Primary endpoint \\
\hline Heidsma et al. [13] & Pancreatic neuroendocrine tumor & $\begin{array}{l}\text { Pancreatoduodenectomy, distal pan- } \\
\text { createctomy, enucleation }\end{array}$ & $\begin{array}{l}\text { Incidence of TO, influence on DFS; TO } \\
\text { and different surgical approaches }\end{array}$ \\
\hline Mehta et al. [22] & Malignant tumor of pancreas or liver & $\begin{array}{l}\text { Minor or major liver resection, minor } \\
\text { or major pancreatic resection }\end{array}$ & $\begin{array}{l}\text { Incidence of TO, TO rates according to } \\
\text { hospital type }\end{array}$ \\
\hline Merath et al. [17] & ICC & Hepatectomy & $\begin{array}{l}\text { Incidence of TO, factors associated } \\
\text { with TO, TO rates according to } \\
\text { hospital type }\end{array}$ \\
\hline Merath et al. [23] & $\begin{array}{l}\text { All indications for mentioned proce- } \\
\text { dure }\end{array}$ & $\begin{array}{l}\text { Minor or major liver resection, minor } \\
\text { or major pancreatic resection }\end{array}$ & $\begin{array}{l}\text { Incidence of TO, factors associated } \\
\text { with TO, TO and different surgical } \\
\text { approaches }\end{array}$ \\
\hline Sweigert et al. [20] & Pancreatic adenocarcinoma & Pancreatoduodenectomy & $\begin{array}{l}\text { Incidence of TO, factors associated } \\
\text { with TO, influence on OS }\end{array}$ \\
\hline Tsilimigras et al. [19] & $\mathrm{HCC}, \mathrm{ICC}$ & Hepatectomy & $\begin{array}{l}\text { Incidence of TO, factors associated } \\
\text { with TO, influence on OS }\end{array}$ \\
\hline Van Roessel et al. [24] & $\begin{array}{l}\text { All indications for mentioned proce- } \\
\text { dure }\end{array}$ & $\begin{array}{l}\text { Pancreatoduodenectomy, distal pan- } \\
\text { createctomy }\end{array}$ & $\begin{array}{l}\text { Incidence of TO, factors associated } \\
\text { with TO, TO rates according to } \\
\text { hospital type }\end{array}$ \\
\hline
\end{tabular}

TO textbook outcome, DFS disease-free survival, HCC hepatocellular cholangiocarcinoma, ICC intrahepatic cholangiocarcinoma, $O S$ overall survival

114 patients $(40 \%)$ were diagnosed with cholangitis preoperatively. Most patients underwent biliary drainage $(86 \%$, $n=243$ ) before surgery. Portal vein embolization was part of the preoperative workup in $46 \%(n=129)$ of patients, all receiving right-sided resections. The majority of patients underwent right-sided liver resection $(63 \%, n=178)$ of which $58 \%(n=165)$ were right trisectionectomies. Thirtyseven percent $(n=105)$ underwent left-sided liver resection, of which $23 \%$ ( $n=64)$ were left trisectionectomies. En bloc portal vein resection was performed in $57 \%(n=162)$ of cases.

\section{Postoperative morbidity, mortality, and textbook outcome}

Most patients suffered from postoperative complications (90\%, $n=254)$, while major complications (grade IIIa-V) occurred in 66\% $(n=186)$ [21]. Common complications were infection $(53 \%, n=150)$, pleural effusion $(34 \%$, $n=96)$, bile leakage $(30 \%, n=86)$, and postoperative liver failure $(26 \%, n=74)$. Supplementary Table $S 1$ gives an overview of the specific postoperative complications. After resection, the median length of hospital stay was 23 (3-213) days. The 30-day and 90-day mortality were 8\% $(n=22)$ and $15 \%(n=42)$. TO could be achieved in $24 \%$ $(n=67)$, severe postoperative complications where the main reason not to be included in the TO group. From all patients, $22 \%$ ( $n=61)$ had to be readmitted or had a prolonged hospital stay $(25 \%, n=72)$, thus not meeting the TO criteria. Some differences in patients' characteristics were noted among patients who achieved TO versus patients who did not (Table 4). Patients with TO showed lower preoperative CA 19-9 levels (53 kU/1 vs. 95 kU/1, $p=0.047$ ) and did less frequently undergo preoperative biliary drainage $(73 \%$ vs. $90 \%, p=0.001)$. Left-sided hepatectomy was also associated with a higher TO rate when compared to right hepatectomy ( $52 \%$ vs. $32 \%, p=0.003)$. With regard to right-sided resections only side and extent of resection, TO is achieved in 18\% ( $n=29)$ of right trisectionectomies versus $23 \%(n=3)$ after standard major hepatectomy $(p=0.619)$. For left-sided resections, TO is achieved in 33\% $(n=21)$ after left trisectionectomy vs. $34 \%$ after standard major hepatectomy $(n=14, p=0.888)$.

\section{Binary logistic regression analysis of factors influencing textbook outcome}

To detect independent factors influencing TO, variables shown in Table 5 that showed significant influence on TO in univariable analysis were included in a binary regression analysis model (Table 5). The regression analysis identified preoperative biliary drainage $(\mathrm{OR}=0.405,95 \% \mathrm{CI}$ : $0.194-0.845, p=0.016)$ and left-sided-resection $(\mathrm{OR}=1.899$, 95\% CI: $1.048-3.440, p=0.035)$, as independent factors influencing TO, whereas left-sided resection was associated with higher and preoperative biliary drainage with lower odds of TO. Despite significant differences in univariable analysis, tumor differentiation was not an independent predictor for TO $(\mathrm{OR}=0.547,95 \% \mathrm{CI}$ : 0.263-1.137, $p=0.106)$. The patient-related factors age, sex, and ASA score had no impact on TO (age: $p=0.392$, sex: $p=0.456$, ASA: $p=0.714$ ). 
Table 3 Patient characteristics

\begin{tabular}{|c|c|}
\hline & $\begin{array}{l}\text { Resected perihilar } \\
\text { cholangiocarcinoma } \\
n=283\end{array}$ \\
\hline Age $^{1}$ & $65(33-86)$ \\
\hline $\mathrm{BMI}^{1}$ & $24.6(16.0-38.3)$ \\
\hline Gender $(\text { male })^{2}$ & $171(60)$ \\
\hline \multicolumn{2}{|l|}{ ASA score ${ }^{2}$} \\
\hline 1 & $13(5)$ \\
\hline 2 & $159(56)$ \\
\hline 3 & $107(38)$ \\
\hline 4 & $4(1)$ \\
\hline \multicolumn{2}{|c|}{ Bismuth-Corlette $^{2}$} \\
\hline I & $13(5)$ \\
\hline II & $20(7)$ \\
\hline IIIa & $72(26)$ \\
\hline IIIb & $55(20)$ \\
\hline IV & $116(42)$ \\
\hline \multicolumn{2}{|l|}{ UICC stage $^{2}$} \\
\hline I & $12(4)$ \\
\hline II & $100(36)$ \\
\hline IIIa & $32(12)$ \\
\hline IIIb & $127(46)$ \\
\hline IVa & $7(2)$ \\
\hline $\mathrm{IVb}$ & $0(0)$ \\
\hline \multicolumn{2}{|c|}{ Resection margin $^{2}$} \\
\hline R0 & $189(68)$ \\
\hline $\mathrm{R} 1$ & $89(32)$ \\
\hline \multicolumn{2}{|c|}{ Lymph node status ${ }^{2}$} \\
\hline No & $146(53)$ \\
\hline $\mathrm{N}+$ & $132(48)$ \\
\hline \multicolumn{2}{|c|}{ Microvascular invasion $^{2}$} \\
\hline Yes & $48(19)$ \\
\hline No & $206(81)$ \\
\hline \multicolumn{2}{|c|}{ Histopathological grading $^{2}$} \\
\hline Grade 1 & $15(5)$ \\
\hline Grade 2 & $190(68)$ \\
\hline Grade 3 & $73(26)$ \\
\hline \multicolumn{2}{|c|}{ Perineural sheath infiltration ${ }^{2}$} \\
\hline Yes & $207(88)$ \\
\hline No & $28(12)$ \\
\hline \multicolumn{2}{|c|}{ Lymphangitis carcinomatosa $^{2}$} \\
\hline Yes & $100(41)$ \\
\hline No & 147 (59) \\
\hline \multicolumn{2}{|l|}{ T stage ${ }^{2}$} \\
\hline is & $1(0)$ \\
\hline 1 & $19(7)$ \\
\hline $2 \mathrm{a}$ & 81 (29) \\
\hline $2 b$ & 94 (33) \\
\hline 3 & 81 (29) \\
\hline 4 & $7(3)$ \\
\hline
\end{tabular}

Table 3 (continued)

\begin{tabular}{|c|c|}
\hline & $\begin{array}{l}\text { Resected perihilar } \\
\text { cholangiocarcinoma } \\
n=283\end{array}$ \\
\hline \multicolumn{2}{|l|}{ Preoperative biliary drainage $^{2}$} \\
\hline Yes & $243(86)$ \\
\hline No & $40(14)$ \\
\hline \multicolumn{2}{|l|}{ Portal vein embolization ${ }^{2}$} \\
\hline Yes & $129(46)$ \\
\hline No & $154(54)$ \\
\hline \multicolumn{2}{|l|}{ Preoperative cholangitis $^{2}$} \\
\hline Yes & $114(40)$ \\
\hline No & $169(60)$ \\
\hline \multicolumn{2}{|l|}{ Resection side $^{2}$} \\
\hline Left hepatectomy & $105(37)$ \\
\hline Extended left hepatectomy & $41(15)$ \\
\hline Left trisectionectomy & $64(23)$ \\
\hline Right hepatectomy & $178(63)$ \\
\hline Extended right hepatectomy & $13(5)$ \\
\hline Right trisectionectomy & $165(58)$ \\
\hline \multicolumn{2}{|l|}{ Portal vein resection ${ }^{2}$} \\
\hline Yes & $162(57)$ \\
\hline No & $121(43)$ \\
\hline Operating time (min) & $385(112-849)$ \\
\hline \multicolumn{2}{|l|}{ Complications (Clavien-Dindo) ${ }^{2}$} \\
\hline None & $29(10)$ \\
\hline I & $12(4)$ \\
\hline II & $56(20)$ \\
\hline IIIa & $84(30)$ \\
\hline IIIb & $51(18)$ \\
\hline IVa & $6(2)$ \\
\hline $\mathrm{IVb}$ & $1(0)$ \\
\hline $\mathrm{V}$ & $44(16)$ \\
\hline Severe complications (grade IIIa-V) & $186(66)$ \\
\hline Preoperative ALAT (U/l) & $66(9-1270)$ \\
\hline Preoperative ASAT(U/l) & $55(13-3352)$ \\
\hline CA $19-9(\mathrm{kU} / \mathrm{l})^{1}$ & $79(1-32670)$ \\
\hline ICU stay (days) ${ }^{1}$ & $4(1-123)$ \\
\hline Hospital stay (days) ${ }^{1}$ & $23(3-213)$ \\
\hline 30-day mortality ${ }^{2}$ & $22(8)$ \\
\hline 90-day mortality ${ }^{2}$ & $42(15)$ \\
\hline Hospital readmission & $61(22)$ \\
\hline Prolonged hospital stay & $72(25)$ \\
\hline \multicolumn{2}{|l|}{ Preoperative chemotherapy } \\
\hline Yes & $10(4)$ \\
\hline No & $273(96)$ \\
\hline \multicolumn{2}{|l|}{ Adjuvant chemotherapy } \\
\hline Yes & $64(23)$ \\
\hline No & $209(77)$ \\
\hline
\end{tabular}

${ }^{1}$ Data is presented as median and range; ${ }^{2}$ Data is presented as count and proportions (\%) 
Table 4 Patient characteristics according to outcome group (TO versus NTO)

\begin{tabular}{|c|c|c|c|}
\hline & $\begin{array}{l}\text { TO } \\
n=67\end{array}$ & $\begin{array}{l}\text { NTO } \\
n=216\end{array}$ & $p$ value \\
\hline Age $^{1}$ & $64(38-81)$ & $65(33-86)$ & 0.696 \\
\hline $\mathrm{BMI}^{1}$ & $24.6(16.0-35.0)$ & $24.9(16.0-38.3)$ & 0.417 \\
\hline Gender $(\text { male })^{2}$ & $37(55)$ & $134(62)$ & 0.319 \\
\hline ASA score ${ }^{2}$ & & & 0.927 \\
\hline 1 & $4(6)$ & $9(4)$ & \\
\hline 2 & $38(57)$ & $121(56)$ & \\
\hline 3 & $24(36)$ & $83(38)$ & \\
\hline 4 & $1(2)$ & $3(1)$ & \\
\hline Bismuth-Corlette $^{2}$ & & & 0.326 \\
\hline I & $3(5)$ & $10(5)$ & \\
\hline II & $2(3)$ & $18(9)$ & \\
\hline IIIa & $14(21)$ & $58(28)$ & \\
\hline IIIb & $17(26)$ & $38(18)$ & \\
\hline IV & $30(46)$ & $86(41)$ & \\
\hline UICC stage ${ }^{2}$ & & & 0.489 \\
\hline I & $4(6)$ & $8(4)$ & \\
\hline II & $27(40)$ & $73(35)$ & \\
\hline IIIa & $6(9)$ & $26(12)$ & \\
\hline IIIb & $27(40)$ & $100(47)$ & \\
\hline IVa & $3(5)$ & $4(2)$ & \\
\hline Resection margin $^{2}$ & & & 0.892 \\
\hline R0 & $46(69)$ & $143(68)$ & \\
\hline $\mathrm{R} 1$ & $21(31)$ & $68(32)$ & \\
\hline Lymph node status ${ }^{2}$ & & & 0.284 \\
\hline No & $39(58)$ & $107(51)$ & \\
\hline $\mathrm{N}+$ & $28(42)$ & $104(49)$ & \\
\hline Microvascular invasion $^{2}$ & & & 0.378 \\
\hline Yes & $9(15)$ & $39(20)$ & \\
\hline No & $51(85)$ & $155(80)$ & \\
\hline Histopathological grading ${ }^{2}$ & & & 0.128 \\
\hline Grade 1 & $4(6)$ & $11(5)$ & \\
\hline Grade 2 & $51(77)$ & $139(66)$ & \\
\hline Grade 3 & $11(17)$ & $62(29)$ & \\
\hline Perineural sheath infiltration ${ }^{2}$ & & & 0.347 \\
\hline Yes & $43(84)$ & $164(89)$ & \\
\hline No & $8(16)$ & $20(11)$ & \\
\hline Lymphangitis carcinomatosa $^{2}$ & & & 0.094 \\
\hline Yes & $18(31)$ & $82(57)$ & \\
\hline No & $40(69)$ & $107(43)$ & \\
\hline $\mathrm{T}_{\text {stage }}{ }^{2}$ & & & 0.399 \\
\hline is & $0(0)$ & $1(0)$ & \\
\hline 1 & $6(9)$ & $13(6)$ & \\
\hline $2 \mathrm{a}$ & $18(27)$ & $63(29)$ & \\
\hline $2 \mathrm{~b}$ & $26(39)$ & $68(32)$ & \\
\hline 3 & $14(21)$ & $67(31)$ & \\
\hline 4 & $3(5)$ & $4(2)$ & \\
\hline Preoperative biliary drainage & & & 0.001 \\
\hline Yes & $49(73)$ & $194(90)$ & \\
\hline No & $18(27)$ & $22(10)$ & \\
\hline Portal vein embolization & & & 0.016 \\
\hline
\end{tabular}


Table 4 (continued)

\begin{tabular}{|c|c|c|c|}
\hline & $\begin{array}{l}\text { TO } \\
n=67\end{array}$ & $\begin{array}{l}\text { NTO } \\
n=216\end{array}$ & $p$ value \\
\hline Yes & $22(33)$ & $107(50)$ & \\
\hline No & $45(67)$ & $109(50)$ & \\
\hline Preoperative cholangitis & & & 0.571 \\
\hline Yes & $25(37)$ & $89(41)$ & \\
\hline No & $42(63)$ & $127(59)$ & \\
\hline Resection side ${ }^{2}$ & & & 0.003 \\
\hline Left hepatectomy & $35(52)$ & $70(32)$ & \\
\hline Extended left hepatectomy & $14(21)$ & $27(13)$ & \\
\hline Left trisectionectomy & $21(31)$ & $43(20)$ & \\
\hline Right hepatectomy & $32(49)$ & $146(68)$ & \\
\hline Extended right hepatectomy & $3(5)$ & $10(5)$ & \\
\hline Right trisectionectomy & $29(43)$ & $136(63)$ & \\
\hline Portal vein resection ${ }^{2}$ & & & 0.018 \\
\hline Yes & $30(45)$ & $132(61)$ & \\
\hline No & $37(55)$ & $84(39)$ & \\
\hline Operating time (min) & $375(232-547)$ & $391(112-849)$ & 0.170 \\
\hline Severe complications (grade IIIa-V) & $0(0)$ & $186(86)$ & $<0.001$ \\
\hline Preoperative ALAT $(\mathrm{U} / \mathrm{l})^{1}$ & $68(14-482)$ & $66(9-1270)$ & 0.446 \\
\hline Preoperative ASAT $(\mathrm{U} / \mathrm{l})^{1}$ & $65(17-430)$ & $54(13-3352)$ & 0.919 \\
\hline CA $19-9(\mathrm{kU} / \mathrm{l})^{1}$ & $53(1-32670)$ & $95(1-23049)$ & 0.047 \\
\hline ICU stay (days) ${ }^{1}$ & $2(2-18)$ & $5(1-123)$ & $<0.001$ \\
\hline Hospital stay (days) ${ }^{1}$ & $16(7-37)$ & $29(3-213)$ & $<0.001$ \\
\hline Preoperative chemotherapy & & & 0.300 \\
\hline Yes & $1(2)$ & $9(4)$ & \\
\hline No & $66(98)$ & $207(96)$ & \\
\hline Adjuvant chemotherapy & & & 0.237 \\
\hline Yes & $18(29)$ & $46(22)$ & \\
\hline No & $44(71)$ & $165(78)$ & \\
\hline
\end{tabular}

${ }^{1}$ Data is presented as median and range; ${ }^{2}$ Data is presented as count and proportions (\%)

\section{Survival analyses}

Median OS of all patients was 29 (24-35) months after resection, whereas DFS was 22 (17-26) months. After excluding patients who died within 90 days after resection, no significant difference between the two groups in either OS $(p=0.280)$ or DFS $(p=0.735)$ analysis could be detected. However, a trend towards better overall survival in patients with TO especially in the late course could be shown that failed to reach statistical significance. Subgroup analyses of patients surviving at least 30 months (Supplementary Table S2) after resection showed better overall survival in patients with TO compared to patients without TO ( 92 versus $60, p=0.039$ ). There was no difference in DFS between patients surviving at least 30 months $(p=0.270$, Figure $2 \mathrm{~A}-\mathrm{D})$.

\section{Discussion}

In recent years, the concept of the TO as a quality measure depicting the ideal surgical outcome has been used in complex oncological surgery, especially in the field of HBP surgery. A recently published multi-center study by Mueller et al. has examined the outcome after PHC surgery for socalled benchmark cases [25]. This study marks an important milestone in the field of PHC surgery since it provides a definition of what is to be considered a benchmark case. However, this is the first study dealing with TO definitions in PHC patients undergoing major hepatectomy. We were able to identify relevant studies dealing with TO in the field of HBP surgery and proposed a TO definition for PHC patients. Furthermore, we were able to identify left hepatectomy as a factor that was independently associated with TO. 
Table 5 Univariable and multivariable analysis of factors influencing textbook outcome in all resected patients

\begin{tabular}{llllll}
\hline All patients $(n=283)$ & & & & \\
\hline Variable & Univariable & & Multivariable & \\
\cline { 2 - 3 } \cline { 5 - 6 } & OR $(95 \%$ CI $)$ & $p$ value & OR (95\% CI) & $p$ value \\
\hline Age & $0.993(0.968-1.018)$ & 0.563 & & $0.989(0.963-1.015)$ & 0.392 \\
Gender (male) & $0.755(0.434-1.314)$ & 0.320 & & $0.800(0.444-1.440)$ & 0.456 \\
Body mass index (kg/m $\left.{ }^{2}\right)$ & $0.960(0.892-1.034)$ & 0.282 & & \\
ASA (>2) & $0.900(0.511-1.583)$ & 0.714 & & \\
Preoperative ALAT (U/l) & $1.000(0.998-1.002)$ & 0.825 & & \\
Preoperative ASAT (U/l) & $0.999(0.997-1.001)$ & 0.457 & & \\
T stage (>2b) & $0.694(0.374-1.290)$ & 0.248 & & \\
UICC (>IIIA) & $0.834(0.480-1.449)$ & 0.520 & & \\
Preoperative CA 19-9 (kU/l) & $1.000(1.000-1.000)$ & 0.999 & & \\
Preoperative drainage (yes) & $0.309(0.154-0.620)$ & 0.001 & & $0.405(0.194-0.845)$ & 0.016 \\
Resection side (left-sided resection) & $2.281(1.306-3.984)$ & 0.004 & & $1.899(1.048-3.440)$ & 0.035 \\
Preoperative cholangitis (yes) & $0.849(0.483-1.494)$ & 0.571 & & \\
Histopathological grading (>G2) & $0.484(0.237-0.986)$ & 0.046 & $0.547(0.263-1.137)$ & 0.106 \\
Perineural sheath infiltration (Pn1) & $0.655(0.270-1.590)$ & 0.350 & & \\
Lymphovascular invasion (L1) & $0.587(0.314-1.098)$ & 0.096 & & \\
Microvascular invasion (V1) & $0.701(0.318-1.547)$ & 0.379 & & \\
Lymph node status (N+) & $0.739(0.424-1.287)$ & 0.285 & & \\
\hline & & & & \\
\hline
\end{tabular}

TO was defined as the absence of 90-day mortality and major complications (i.e., > grade II according to DindoClavien), no hospital readmission within 90 days after discharge, and no prolonged hospital stay (i.e., <75. percentile). According to the literature review, the exclusion criteria for TO were 90-day mortality, severe complications ( $\geq$ IIIa according to Dindo-Clavien), unplanned readmission, and prolonged hospital stay [21]. When it comes to the definition of postoperative mortality, studies differ greatly, as some used 30-day mortality, others 90-day mortality [13, $17,22,23,26]$. Defining postoperative mortality as 30-day mortality may lead to an underestimation of the actual perioperative mortality after liver resection by up to $50 \%$. After an initial steep increase in postoperative mortality, a more or less constant mortality rate is observed after 90 days [5, 27]. Indicators of postoperative morbidity reported in the analyzed TO studies were length of hospital stay (LOS), grade of complications, or specific complications. There is a known high correlation between LOS and incidence of complications [18].

Nevertheless, there is a potential bias when only reporting $\operatorname{LOS}[18,20]$. LOS may not necessarily represent a surgical quality measure, as it may be prolonged by external factors such as inadequately ensured home care at discharge and not morbidity-related [18] or cultural aspects $[5,17]$. For these reasons, a second morbidity measure was added to the "prolonged hospital stay" parameter in the present work. Specific complications to define TO as used by van Roessel et al. were not used because patients often develop more than one complication. Instead of using "no morbidity" as a measure [23], major complications ( $\geq$ IIIa according to Dindo-Clavien) were chosen as a parameter in this analysis, because major complications are frequently observed after MH for PHC [5, 21-23, 26]. Although R0 status was part of the TO definition in half of the TO studies [13, 17, 19, 20], we decided not to include it in the definition of TO in PHC surgery for several reasons. First, the perihilar region is narrow and $\mathrm{R} 1$ resection is not uncommon in PHC surgery [28-30] and patients benefit from surgical resection even in case $\mathrm{R} 1$ status is obtained. Second, especially in the subset of lymph nodepositive patients which counts up to almost $50 \%$, survival is independent of R status [30]. Third, PHC surgery is characterized by high perioperative morbidity and mortality. Thus, TO should rather be defined by an uneventful postoperative course than histopathological criteria.

Overall, a TO rate of $24 \%(n=67)$ was observed in the present study. This is consistent with TO rates obtained after surgical procedures in the field of HBP surgery [17, 19, 20]. Opposite to the findings in other studies [17, 19], age was not found to be influencing TO in the present study. Poor histologic differentiation had a negative impact on the development of a TO in the present study. In general, poor grading is considered a risk for early tumor recurrence and decreased OS [3, 31-33]. Additionally, however, these factors may also reflect an aggressive tumor biology indicating advanced tumor disease. This is related to frailty, which leads to increased morbidity and mortality [34]. 
A

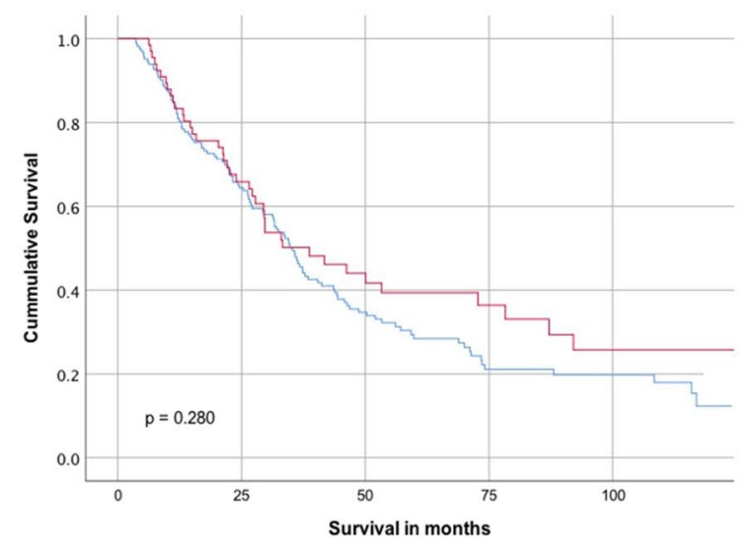

C

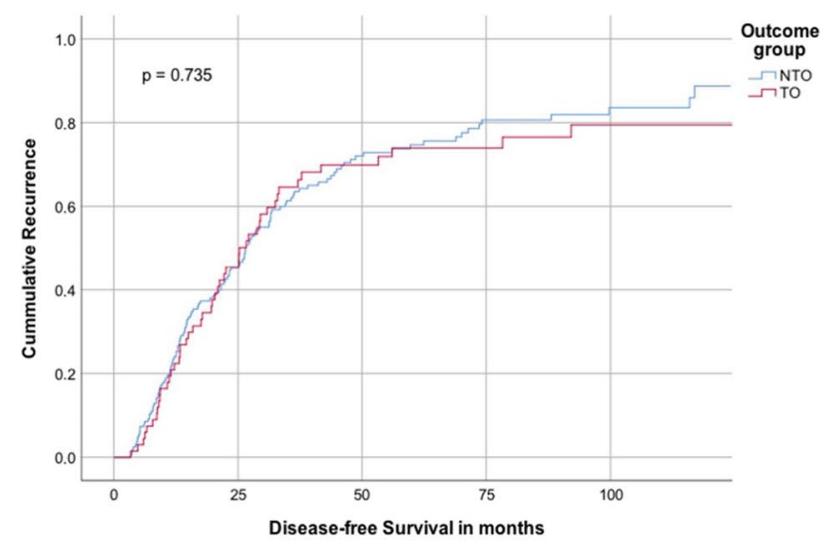

Fig. 2 Overall survival and disease-free survival according to outcome group. Kaplan-Meier curves of (A) overall survival of all resected patients with perihilar cholangiocarcinoma excluding 90-day mortality according to outcome group; (B) overall survival of all resected patients surviving $>30$ months according to outcome group;

In the present work, left hepatectomy could be identified as an independent factor favoring TO which is in line with another study [19] compared with right hepatectomy. This is due to a significantly smaller future liver remnant (FLR), which is a risk factor for PHLF which consecutively leads to increased postoperative mortality rates $[7,35]$

As a second independent factor influencing TO, we found that preoperative biliary drainage to be associated with not achieving TO. Similar to the results of this work, Zhang et al. showed a significantly increased morbidity rate in patients with biliary drainage [36]. This is likely attributable to advanced disease as well as preoperative cholangitis; thus, drainage should be considered a surrogate parameter for advanced disease.

The median overall survival in the current study in the overall cohort was 29 (24-35) months, which is in line with other studies (13-40 months) [10, 28, 29, 37, 38]. Some previous studies in the field of HBP surgery have shown
B

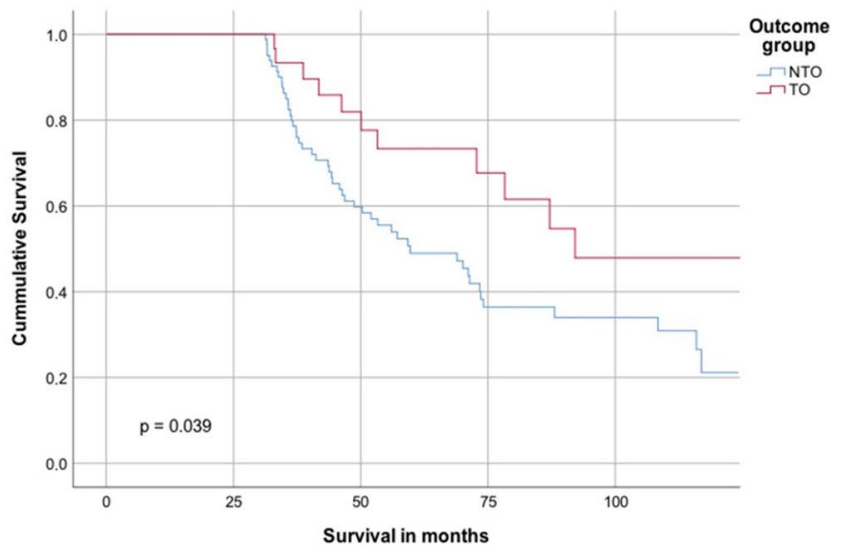

D

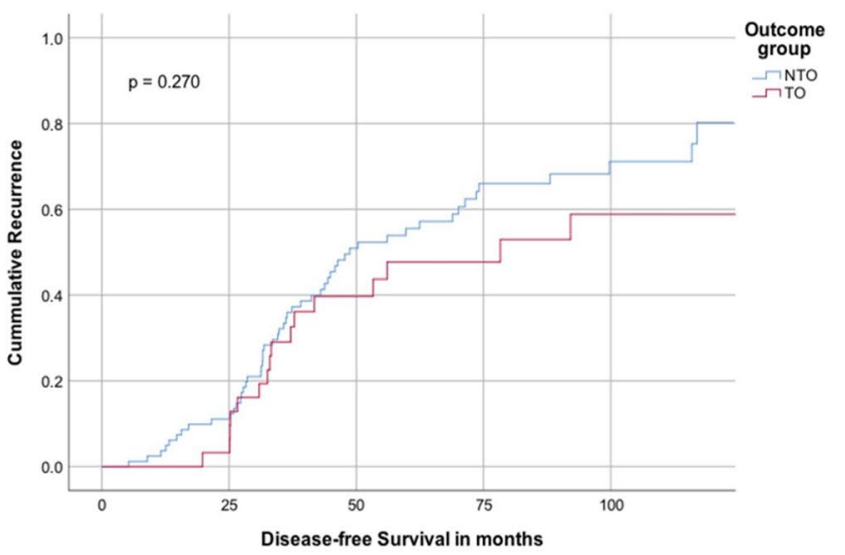

(C) disease-free survival of all resected patients with perihilar cholangiocarcinoma excluding 90-day mortality according to outcome group; and (D) disease-free survival of all resected patients with perihilar cholangiocarcinoma surviving $>30$ months according to outcome group

improved OS and DFS if TO is achieved [13, 19, 20, 39]. There was no significant difference in survival between the TO and NTO groups. However, in the long-term (beginning from month 30), there was a clear trend towards a better OS and DFS in the TO group compared to the NTO group. Reasons for improved long-term survival when TO is achieved may be a timely connection to adjuvant chemotherapy. This can be delayed or not occur at all in patients with major complications. In this work, a higher number of patients in the TO group tended to receive adjuvant chemotherapy in the overall cohort which can have a positive effect on OS and DFS [40]. However, it is difficult to draw final conclusions since exact follow-up data including the applied chemotherapy regimen, dose, and information on, e.g., premature termination of adjuvant chemotherapy due to side effects are missing in a relevant proportion of patients. In general, recommendations for adjuvant chemotherapy included either gemcitabine ( \pm cisplatin) or fluorouracil/capecitabine. 
There are several limitations in the present study. First, it is a retrospective analysis which can lead to bias. Second, clear definitions of TO in PHC lack, so the comparability is limited. However, this is the first study proposing a definition of TO in patients with PHC undergoing MH based on a thorough literature review, which is evaluated and tested on a large cohort of patients.

\section{Conclusions}

TO is a quality indicator that is not exclusively limited to reporting mortality, but more comprehensively represents a desired postoperative course. In the present study, we introduced the composite quality measure TO into PHC. This is of great importance in order to allow a comparison between studies or centers. Therefore, there is a great need for a uniform and robust TO definition in future studies. We identified left hepatectomy as an independent factor positively influencing TO. In cases with central tumors, where both right- and left-sided resections are feasible, this underlines the importance of a careful selection of patients who are scheduled for right hepatectomy, which are superior in terms of oncological radicality but associated with higher postoperative morbidity and mortality.

Supplementary Information The online version contains supplementary material available at https://doi.org/10.1007/s00423-022-02467-y.

Funding Open Access funding enabled and organized by Projekt DEAL.

\section{Declarations}

Competing interests The authors declare no competing interests.

Open Access This article is licensed under a Creative Commons Attribution 4.0 International License, which permits use, sharing, adaptation, distribution and reproduction in any medium or format, as long as you give appropriate credit to the original author(s) and the source, provide a link to the Creative Commons licence, and indicate if changes were made. The images or other third party material in this article are included in the article's Creative Commons licence, unless indicated otherwise in a credit line to the material. If material is not included in the article's Creative Commons licence and your intended use is not permitted by statutory regulation or exceeds the permitted use, you will need to obtain permission directly from the copyright holder. To view a copy of this licence, visit http://creativecommons.org/licenses/by/4.0/.

\section{References}

1. Razumilava N, Gores GJ (2014) Cholangiocarcinoma. Lancet 383(9935):2168-2179. https://doi.org/10.1016/s0140-6736(13) 61903-0
2. Neuhaus P, Thelen A, Jonas S, Puhl G, Denecke T, VeltzkeSchlieker W, Seehofer D (2012) Oncological superiority of hilar en bloc resection for the treatment of hilar cholangiocarcinoma. Ann Surg Oncol 19(5):1602-1608. https://doi.org/10.1245/ s10434-011-2077-5

3. Neuhaus P, Jonas S, Bechstein WO, Lohmann R, Radke C, Kling N, Wex C, Lobeck H, Hintze R (1999) Extended resections for hilar cholangiocarcinoma. Ann Surg 230(6):808-818; discussion 819. https://doi.org/10.1097/00000658-199912000-00010

4. Franken LC, Rassam F, van Lienden KP, Bennink RJ, Besselink MG, Busch OR, Erdmann JI, van Gulik TM, Olthof PB (2020) Effect of structured use of preoperative portal vein embolization on outcomes after liver resection of perihilar cholangiocarcinoma. BJS Open 4(3):449-455. https://doi.org/10.1002/bjs5.50273

5. Franken LC, Schreuder AM, Roos E, van Dieren S, Busch OR, Besselink MG, van Gulik TM (2019) Morbidity and mortality after major liver resection in patients with perihilar cholangiocarcinoma: a systematic review and meta-analysis. Surgery 165(5):918-928. https://doi.org/10.1016/j.surg.2019.01.010

6. Olthof PB, Aldrighetti L, Alikhanov R, Cescon M, Groot Koerkamp B, Jarnagin WR, Nadalin S, Pratschke J, Schmelze M, Sparrelid E, Lang H, Guglielmi A, van Gulik TM (2020) Portal vein embolization is associated with reduced liver failure and mortality in high-risk resections for perihilar cholangiocarcinoma. Ann Surg Oncol 27(7):2311-2318. https://doi.org/10.1245/ s10434-020-08258-3

7. Ribero D, Zimmitti G, Aloia TA, Shindoh J, Forchino F, Amisano M, Passot G, Ferrero A, Vauthey J-N (2016) Preoperative cholangitis and future liver remnant volume determine the risk of liver failure in patients undergoing resection for hilar cholangiocarcinoma. J Am Coll Surg 223(1):87-97. https://doi.org/10.1016/j. jamcollsurg.2016.01.060

8. Lee EC, Park SJ, Han SS, Shim JR, Park HM, Lee SD, Kim SH (2018) Risk prediction of post-hepatectomy liver failure in patients with perihilar cholangiocarcinoma. J Gastroenterol Hepatol 33(4):958-965. https://doi.org/10.1111/jgh.13966

9. Yokoyama Y, Nagino M, Nimura Y (2007) Mechanism of impaired hepatic regeneration in cholestatic liver. J HepatoBiliary-Pancreat Surg 14(2):159-166. https://doi.org/10.1007/ s00534-006-1125-1

10. Olthof PB, Coelen RJS, Wiggers JK, Besselink MGH, Busch ORC, van Gulik TM (2016) External biliary drainage following major liver resection for perihilar cholangiocarcinoma: impact on development of liver failure and biliary leakage. HPB 18(4):348353. https://doi.org/10.1016/j.hpb.2015.11.007

11. Baton O, Azoulay D, Adam DVR, Castaing D (2007) Major hepatectomy for hilar cholangiocarcinoma type 3 and 4: prognostic factors and longterm outcomes. J Am Coll Surg 204(2):250-260. https://doi.org/10.1016/j.jamcollsurg.2006.10.028

12. Nagino M, Kamiya J, Uesaka K, Sano T, Yamamoto H, Hayakawa N, Kanai M, Nimura Y (2001) Complications of hepatectomy for hilar cholangiocarcinoma. World J Surg 25(10):1277-1283. https://doi.org/10.1007/s00268-001-0110-8

13. Heidsma CM, Hyer M, Tsilimigras DI, Rocha F, Abbott DE, Fields R, Smith PM, Poultsides GA, Cho C, Maithel SK, Pawlik TM (2020) Incidence and impact of textbook outcome among patients undergoing resection of pancreatic neuroendocrine tumors: results of the US Neuroendocrine Tumor Study Group. J Surg Oncol 121(8):1201-1208. https://doi.org/10.1002/jso.25900

14. Salet N, Bremmer RH, Verhagen M, Ekkelenkamp VE, Hansen $\mathrm{BE}$, de Jonge PJF, de Man RA (2018) Is textbook outcome a valuable composite measure for short-term outcomes of gastrointestinal treatments in the Netherlands using hospital information system data? A retrospective cohort study. BMJ Open 8(2):e019405. https://doi.org/10.1136/bmjopen-2017-019405 
15. Denbo J, Anaya DA (2020) Textbook outcomes following liver resection for cancer: a new standard for quality benchmarking and patient decision making. Ann Surg Oncol. https://doi.org/10.1245/ s10434-020-08550-2

16. Busweiler LA, Schouwenburg MG, van Berge Henegouwen MI, Kolfschoten NE, de Jong PC, Rozema T, Wijnhoven BP, van Hillegersberg R, Wouters MW, van Sandick JW (2017) Textbook outcome as a composite measure in oesophagogastric cancer surgery. Br J Surg 104(6):742-750. https://doi.org/10.1002/bjs.10486

17. Merath K, Chen Q, Bagante F, Alexandrescu S, Marques HP, Aldrighetti L, Maithel SK, Pulitano C, Weiss MJ, Bauer TW, Shen F, Poultsides GA, Soubrane O, Martel G, Koerkamp BG, Guglielmi A, Itaru E, Cloyd JM, Pawlik TM (2019) A multi-institutional international analysis of textbook outcomes among patients undergoing curative-intent resection of intrahepatic cholangiocarcinoma. JAMA Surg 154(6):e190571. https://doi.org/10.1001/ jamasurg.2019.0571

18. Aiken T, Abbott DE (2020) Textbook oncologic outcome: a promising summary metric of high-quality care, but are we on the same page? J Surg Oncol 121(6):923-924. https://doi.org/ $10.1002 /$ jso. 25872

19. Tsilimigras DI, Sahara K, Moris D, Mehta R, Paredes AZ, Ratti F, Marques HP, Soubrane O, Lam V, Poultsides GA, Popescu I, Alexandrescu S, Martel G, Workneh A, Guglielmi A, Hugh T, Aldrighetti L, Weiss M, Bauer TW et al (2020) Assessing textbook outcomes following liver surgery for primary liver cancer over a 12-year time period at major hepatobiliary centers. Ann Surg Oncol. https://doi.org/10.1245/s10434-020-08548-w

20. Sweigert PJ, Eguia E, Baker MS, Paredes AZ, Tsilimigras DI, Dillhoff M, Ejaz A, Cloyd J, Tsung A, Pawlik TM (2020) Assessment of textbook oncologic outcomes following pancreaticoduodenectomy for pancreatic adenocarcinoma. J Surg Oncol 121(6):936-944. https://doi.org/10.1002/jso.25861

21. Dindo D, Demartines N, Clavien PA (2004) Classification of surgical complications: a new proposal with evaluation in a cohort of 6336 patients and results of a survey. Ann Surg 240(2):205-213. https://doi.org/10.1097/01.sla.0000133083. 54934.ae

22. Mehta R, Tsilimigras DI, Paredes AZ, Sahara K, Dillhoff M, Cloyd JM, Ejaz A, White S, Pawlik TM (2020) Dedicated cancer centers are more likely to achieve a textbook outcome following hepatopancreatic surgery. Ann Surg Oncol 27(6):18891897. https://doi.org/10.1245/s10434-020-08279-y

23. Merath K, Chen Q, Bagante F, Beal E, Akgul O, Dillhoff M, Cloyd JM, Pawlik TM (2020) Textbook outcomes among medicare patients undergoing hepatopancreatic surgery. Ann Surg 271(6):1116-1123. https://doi.org/10.1097/sla.0000000000 003105

24. van Roessel S, Mackay TM, van Dieren S, van der Schelling GP, Nieuwenhuijs VB, Bosscha K, van der Harst E, van Dam RM, Liem MSL, Festen S, Stommel MWJ, Roos D, Wit F, Molenaar IQ, de Meijer VE, Kazemier G, de Hingh I, van Santvoort HC, Bonsing BA et al (2020) Textbook outcome: nationwide analysis of a novel quality measure in pancreatic surgery. Ann Surg 271(1):155-162. https://doi.org/10.1097/sla.0000000000 003451

25. Mueller M, Breuer E, Mizuno T, Bartsch F, Ratti F, Benzing C, Ammar-Khodja N, Sugiura T, Takayashiki T, Hessheimer A, Kim HS, Ruzzenente A, Ahn KS, Wong T, Bednarsch J, D'Silva M, Koerkamp BG, Jeddou H, López-López V et al (2021) Perihilar cholangiocarcinoma - novel benchmark values for surgical and oncological outcomes from 24 expert centers. Ann Surg 274(5):780-788. https://doi.org/10.1097/sla.00000 00000005103
26. Mehta R, Paredes AZ, Tsilimigras DI, Moro A, Sahara K, Farooq A, Dillhoff M, Cloyd JM, Tsung A, Ejaz A, Pawlik TM (2020) Influence of hospital teaching status on the chance to achieve a textbook outcome after hepatopancreatic surgery for cancer among Medicare beneficiaries. Surgery 168(1):92-100. https://doi.org/10.1016/j.surg.2020.02.024

27. Mayo SC, Shore AD, Nathan H, Edil BH, Hirose K, Anders RA, Wolfgang CL, Schulick RD, Choti MA, Pawlik TM (2011) Refining the definition of perioperative mortality following hepatectomy using death within 90 days as the standard criterion. HPB (Oxford) 13(7):473-482. https://doi.org/10.1111/j. 1477-2574.2011.00326.x

28. Ebata T, Mizuno T, Yokoyama Y, Igami T, Sugawara G, Nagino M (2018) Surgical resection for Bismuth type IV perihilar cholangiocarcinoma. Br J Surg 105(7):829-838. https://doi.org/10. 1002/bjs. 10556

29. Nagino M, Ebata T, Yokoyama Y, Igami T, Sugawara G, Takahashi Y, Nimura Y (2013) Evolution of surgical treatment for perihilar cholangiocarcinoma: a single-center 34-year review of 574 consecutive resections. Ann Surg 258(1):129-140. https:// doi.org/10.1097/SLA.0b013e3182708b57

30. Benzing C, Krenzien F, Mieg A, Wolfsberger A, Andreou A, Nevermann N, Pelzer U, Fehrenbach U, Haiden LM, Öllinger R, Schöning W, Schmelzle M, Pratschke J (2021) A tailored approach in lymph node-positive perihilar cholangiocarcinoma. Langenbeck's Arch Surg 406(5):1499-1509. https://doi.org/10. 1007/s00423-021-02154-4

31. Groot Koerkamp B, Wiggers JK, Gonen M, Doussot A, Allen PJ, Besselink MGH, Blumgart LH, Busch ORC, D'Angelica MI, DeMatteo RP, Gouma DJ, Kingham TP, van Gulik TM, Jarnagin WR (2015) Survival after resection of perihilar cholangiocarcinoma-development and external validation of a prognostic nomogram. Ann Oncol 26(9):1930-1935. https://doi.org/10. 1093/annonc/mdv279

32. Tang Z, Yang Y, Zhao Z, Wei K, Meng W, Li X (2018) The clinicopathological factors associated with prognosis of patients with resectable perihilar cholangiocarcinoma: a systematic review and meta-analysis. Medicine (Baltimore) 97(34):e11999. https://doi.org/10.1097/md.0000000000011999

33. Groot Koerkamp B, Wiggers JK, Allen PJ, Besselink MG, Blumgart LH, Busch OR, Coelen RJ, D'Angelica MI, DeMatteo RP, Gouma DJ, Kingham TP, Jarnagin WR, van Gulik TM (2015) Recurrence rate and pattern of perihilar cholangiocarcinoma after curative intent resection. J Am Coll Surg 221(6):10411049. https://doi.org/10.1016/j.jamcollsurg.2015.09.005

34. Ethun CG, Bilen MA, Jani AB, Maithel SK, Ogan K, Master VA (2017) Frailty and cancer: implications for oncology surgery, medical oncology, and radiation oncology. CA Cancer J Clin 67(5):362-377. https://doi.org/10.3322/caac.21406

35. Wiggers JK, Groot Koerkamp B, Cieslak KP, Doussot A, van Klaveren D, Allen PJ, Besselink MG, Busch OR, D'Angelica MI, DeMatteo RP, Gouma DJ, Kingham TP, van Gulik TM, Jarnagin WR (2016) Postoperative mortality after liver resection for perihilar cholangiocarcinoma: development of a risk score and importance of biliary drainage of the future liver remnant. J Am Coll Surg 223(2):321-331.e321. https://doi.org/10.1016/j. jamcollsurg.2016.03.035

36. Zhang XF, Beal EW, Merath K, Ethun CG, Salem A, Weber SM, Tran T, Poultsides G, Son AY, Hatzaras I, Jin L, Fields RC, Weiss M, Scoggins C, Martin RCG, Isom CA, Idrees K, Mogal HD, Shen P et al (2018) Oncologic effects of preoperative biliary drainage in resectable hilar cholangiocarcinoma: percutaneous biliary drainage has no adverse effects on survival. J Surg Oncol 117(6):1267-1277. https://doi.org/10.1002/jso.24945 
37. Sano T, Shimada K, Sakamoto Y, Yamamoto J, Yamasaki S, Kosuge T (2006) One hundred two consecutive hepatobiliary resections for perihilar cholangiocarcinoma with zero mortality. Ann Surg 244(2):240-247. https://doi.org/10.1097/01.sla.00002 17605.66519 .38

38. Bednarsch J, Czigany Z, Lurje I, Tacke F, Strnad P, Ulmer TF, Gaisa NT, Bruners P, Neumann UP, Lurje G (2020) Left- versus right-sided hepatectomy with hilar en-bloc resection in perihilar cholangiocarcinoma. HPB (Oxford) 22(3):437-444. https://doi. org/10.1016/j.hpb.2019.07.003

39. van der Werf LR, Wijnhoven BPL, Fransen LFC, van Sandick JW, Nieuwenhuijzen GAP, Busweiler LAD, van Hillegersberg R, Wouters M, Luyer MDP, van Berge Henegouwen MI (2019) A national cohort study evaluating the association between short-term outcomes and long-term survival after esophageal and gastric cancer surgery. Ann Surg 270(5):868-876. https:// doi.org/10.1097/sla.0000000000003520

40. Primrose JN, Fox RP, Palmer DH, Malik HZ, Prasad R, Mirza D, Anthony A, Corrie P, Falk S, Finch-Jones M, Wasan H, Ross P, Wall L, Wadsley J, Evans JTR, Stocken D, Praseedom R, Ma YT, Davidson B et al (2019) Capecitabine compared with observation in resected biliary tract cancer (BILCAP): a randomised, controlled, multicentre, phase 3 study. Lancet Oncol 20(5):663-673. https://doi.org/10.1016/s1470-2045(18)30915-x

Publisher's note Springer Nature remains neutral with regard to jurisdictional claims in published maps and institutional affiliations. 